\title{
Automatic System of Monitoring and Diagnostics of Sparking in Contact
}

\author{
Sergey Solyonyj ${ }^{1}$, Oksana Solenaya ${ }^{1}$ and Andrey Roznhin ${ }^{1,2}$ \\ ${ }^{1}$ SUAI, 67, Bolshaya Morskaia str., St. Petersburg, 190000, Russia \\ ${ }^{2}$ SPIIRAS 39, 14 line, St. Petersburg, 199178, Russia
}

\begin{abstract}
Most common occurrence of a short circuit is a consequence of a combined impact of various ignition sources - electrical sparking or monotonic alteration of resistance in contact joints - on the low-voltage network. An automatic system of monitoring and diagnostics of electrical wiring in case of an electrical sparking or monotonic alteration of the electrical contact resistance was developed. We suggest two ways of applying the elaborated system: the stationary and the mobile one. The system has two ways of applying: the stationary and the mobile one. It estimates permanently the level of fire safety of the power supply system and promptly form a signal for the abnormal electrical wiring power cut and a signal of its level of fire risk. In addition, the introduced system can serve as a subsystem for providing a controlled level of fire safety in the "Smart house" or "Smart Grid" systems.
\end{abstract}

\section{Introduction}

Up to 1.5 million fires occur yearly worldwide in facilities related to human vital activity [1], meanwhile nearly 30 per cent of them take place due to emergency implications of electric current in electric wiring [2]. There are several main emergency modes of electrical wiring marked out: the short circuit $-80 \%$, electrical sparking, warming or monotonic alteration of electrical contact resistance $-11 \%$, overloads $-3,5 \%$, earth leakage $-1,6 \%$, overvoltage $2,5 \%$, other causes hard to classify $-1,4 \%$. Thus, the emergency events of short circuit prevail, but it is correct not in all cases. As nearly all the electrical wiring is insulated, the short circuit appearance requires preliminary insulation breakdown, which can be caused by action of electrical sparking, warming or monotonic alteration of electrical contact resistance. [3] Today technologies aimed at detection and further analysis of emergency modes of electrical wiring described above are developed and tested. Almost all of them suggest that physically the sparking alarm would be received by means of current sensor, put on the phase wire [4]. Besides the methods using indirect approach to determine the sparking point or warming point of the electrical wiring, the most widespread is the infrared temperature measurement by pyrometers [5]. However, the methods of pyrometry cannot always be applied practically, as not all of the wiring is available for analysis. Moreover, some false conclusions can be made while using these methods, as the strongly loaded phase in the contact joint point without sparking can have the same temperature as with sparking.

A separate part of researches are scientific works on modeling emergency sparking processes and arc formation in contact joints, which allow one to test without any fire risk consequences the materials used for creating wire and cable, contacts and protective and commutative appliances as well as analyze the alteration of basic physical quantities (current, voltage, frequency) in case of sparking [6].

In works [7]-[9] the methods based on Wavelet transform, allowing one to convert the original sparking alarm from time representation to time-and-frequency representation are reviewed. Thus, the physical quantities of the original sparking alarm become more estimable, which allows one to reduce the initial data set for the generalized spectral analysis. These methods allow one to identify the sparking wiring; however, they require high computational and hardware costs.

The methods of determining the impedance of the wiring, suggested in works [10], [11], enable one to estimate the ohmic resistance value of the wiring in terms of nominal and emergency exploitation (due to the alteration of the electrical contact resistance) with a subsequent comparison of the obtained results. Though these methods are attractive, they do not always give adequate convergence of the results of experiments and simulations, as modern electrical loads can change their input impedance in operation.

There are also methods of identifying the sparking alarm, which are based on using morphological filtering [12]. However, they are not suitable for use with alternating current, as they a priori imply that the power system is based on a direct current and no noise is present.

The most promising are the methods that enable one to free from periodic and random interference. They are based on the use of spectral analysis of signals before and after the appearance of sparks in the wiring. This approach is similar to methods of wavelet analysis, but it 
involves the assessment and work with several physical quantities (current, voltage) at a time [13], [14].

Since any electrical wiring is a set of wires and cables, interconnected by different types of contacts and the protection and commutation devices, there is always a chance of accidental damage of the insulation, poor adjustment of contacts, etc. during the installation. Consequently, the initial quality of wiring installation is the key to its future operational and fire safety.

According to the current regulations for the structure of electrical installations [15], the wiring should meet the norms of acceptance tests, due to which the wires and cables, switchgears, panels and wireways of voltage up to $1 \mathrm{kV}$ from distribution points to current collectors shall be tested only for the measurement of the insulation resistance. From this it follows that the current Regulations for the structure of electrical installations do not provide adequate methods and tools for monitoring and diagnostics of electrical wiring on the quality of installation, ageing or carrying out acceptance tests. The principles and approaches outlined in mentioned works allow one to develop an automatic system of monitoring and diagnostics of sparking in contact joints of electrical wiring.

\section{The principle of detection of electrical sparking and contact heating}

The basis of operation of the developed automatic system of monitoring and diagnostics of wiring is the following principle: any electric sparking or monotonic alteration of the electrical contact resistance is accompanied not only by heating, but also by the appearance of load in the operational current, connected to the wiring with a frequency of $50 \mathrm{~Hz}$, high-frequency harmonic components (HHC) (Fig. 1) with a frequency of $500 \mathrm{~Hz}$ and higher [16].

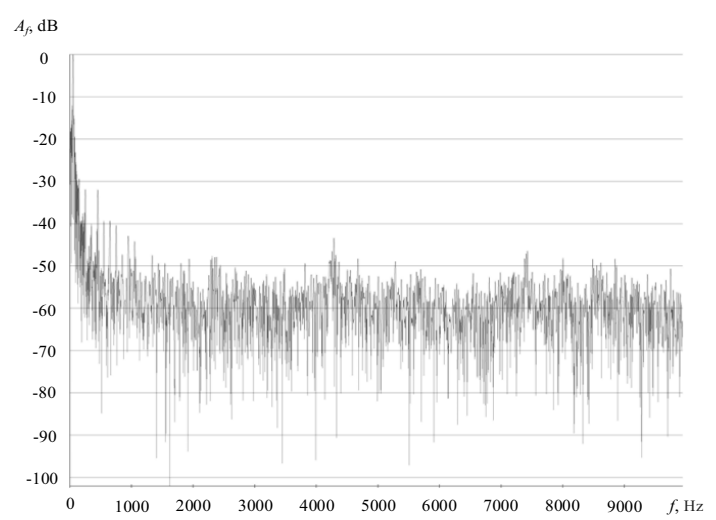

Figure 1. Spectral densities of a signal with a frequency of 50 $\mathrm{Hz}$ with high-frequency harmonic components with a frequency of $500 \mathrm{~Hz}$ and higher

Thus, knowing the duration of the existence of HHC (contact heating) and the duration of pauses between them (contact cooling) it is possible to determine the degree of heating of contact joints, using the averaged experimental curves of heating and cooling of the contact with given load power (Fig. 2). This will allow one to form signals corresponding to the levels of fire risk of existing wiring or that put into operation.

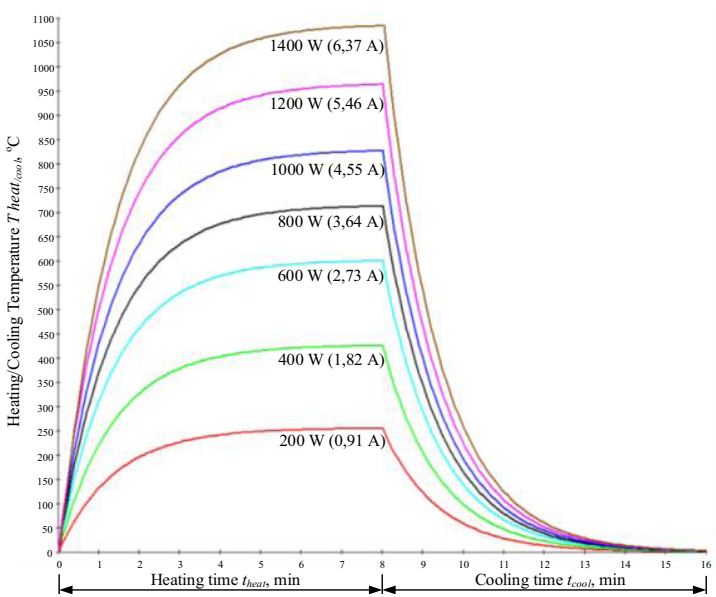

Figure 2. The averaged experimental curves of heating and cooling of the contact with a given load power

It should also be noted that in case of electric sparking, contact resistance $\left(R_{c}\right)$ randomly varies from nearly zero value (best contact) to infinity (no contact). The cooling of the contact occurs in both of these opposite modes; however, with a better contact the joint can cool down maximally to a temperature conditioned by nominal load current and heat maximally when arcing (contact breaking and closing).

Using a digital oscilloscope - a measuring multichannel registering device "RECON-08MC" with a matching unit (shunt SU 10/50), oscillograms of load current with power of $100 \mathrm{~W}$ were recorded while electrical sparking or monotonic alteration of contact resistance. For processing the signals a specialized software was used - a registering archiver support program WinRec-MC, version 2.8.3.0. Fig. 3 represents an oscillogram of load current with power of $100 \mathrm{~W}$ at sparking or monotonic alteration of contact resistance, through which it is connected to the wiring: 1 - section of the best contact ( $R_{c} \approx 0$, rated current flowing through the load); 2 - section of no contact $\left(R_{c} \approx \infty\right.$, current not flowing through the load); 3 - section of resumption of the current flow through the load, accompanied by the transition process (with current increasing more than rated, due to inductive and capacitive nature of the load); 4 - section of electrical sparking or monotonic alteration of contact resistance.

As it can be seen from Fig. 3, heating and cooling of the contact are similar to the heating and cooling of electrical devices in intermittent duty [17], [18]. This mode is characterized by a duty cycle of the electric device, in our case let it be called the length of Contact Sparking (CS):

$$
C S_{C J}=\frac{t_{\text {heat }}}{t_{\text {heat }}+t_{\text {cool }}} \cdot 100, \%
$$

where $t_{\text {heat }}$ is the heating time of the contact (with electrical sparking or monotonic alteration of contact resistance and $R_{c} \approx 0$ ); $t_{\text {cool }}$ - the cooling time (when $R_{c}$ 
$\approx \infty$ and also when $R_{c} \approx 0$ if section 3 is replaced by section 1 (Fig. 3)).

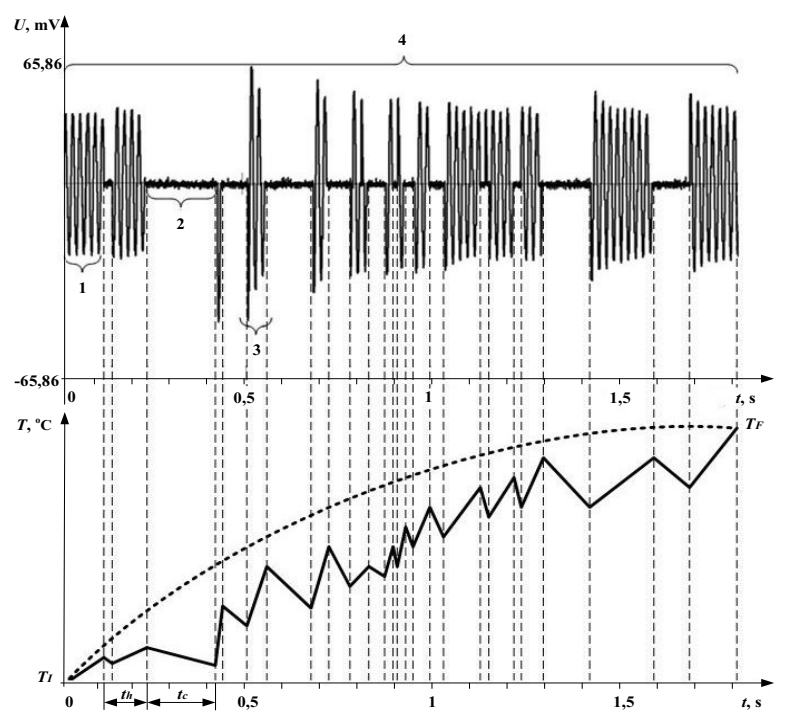

Figure 3. An oscillogram of load current with power of $100 \mathrm{~W}$ at electrical sparking or monotonic alteration of contact resistance and also a schematic graph of its heating/cooling

Thus, in case the electrical sparking or monotonic alteration of the contact resistance occur, the power contact connection begins to heat up from the initial temperature $T_{I}$ (permissible operating temperature of the insulation depending on its class), to a final temperature $T_{F}$, which in some cases may be above the permissible operating temperature of the insulation, i.e. become the ignition temperature.

\section{Flowchart of the automatic system of monitoring and diagnostics of electrical wiring}

The proposed principle of detection of electrical sparking and contact heating was used for development of the automatic system of monitoring and diagnostics of wiring, which is presented in Fig. 4. To start diagnosing the internal electrical wiring must be disconnected from the external through the automatic circuit breaker QF1, whereas the circuit breakers QF2 and QF3 stay on. To the lighting part of the internal wiring, which consists of a junction box 8 and a load-break switch 9, the glow lamps 10 are connected $\left(\cos \varphi=1\right.$ and $\left.P_{L}=100 \mathrm{~W}\right)$ by the number of electrical sockets. The active loads $12(\cos \varphi=$ 1 and $P_{L}=100 \mathrm{~W}$ ) are connected to the female connector of the internal wiring, consisting of a junction box 8 and an electrical receptacle 11 , by the number of receptacles (glow lamps can be used). In case there are multiple varieties of electrical wiring (heating, water heating and other), the active loads are connected to them due to the number of places for electrical sockets and receptacles. A current load sensor 1 of the automatic system of monitoring and diagnostics of electrical wiring 7 is put on the phase wire. After performing the operations mentioned above the automatic circuit breaker QF1 is turned on and the internal wiring is ready for diagnostics.

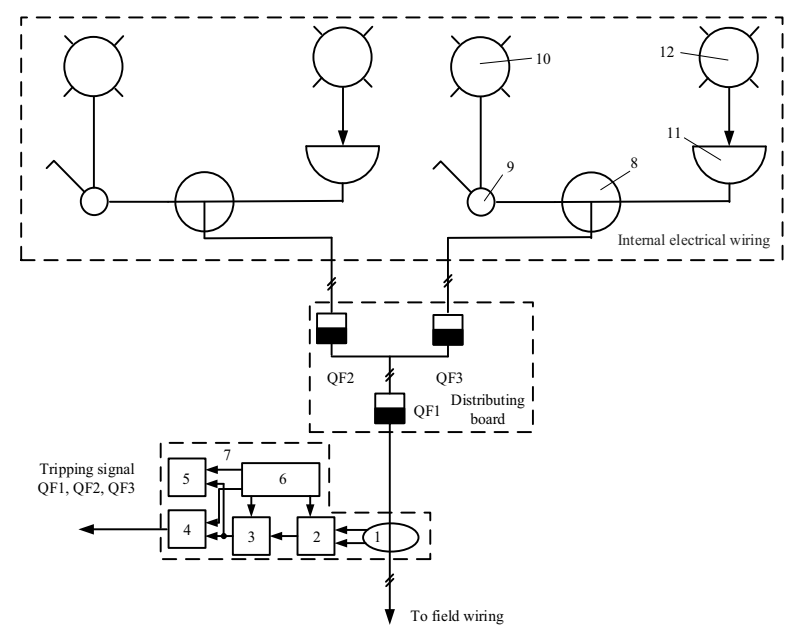

Figure 4. The flowchart of the automatic system of monitoring and diagnostics of electrical wiring

If the installation of internal wiring (wires, cables and contacts) had been conducted poorly (mechanical damage to wires and cables takes place, lose or faulty contacts are present), electrical sparking or monotonic change of contact resistance would appear in these places - the electrical commutation (Fig. 1, 3).

The signal (Fig. 1,3) is taken by means of the current load sensor 1. Next, the signal is sent to HHCs selection box 2 . In block 2 the primary signal enhancement, highfrequency filtration, secondary signal enhancement, automatic offset from periodic and stochastic interference, conditioned by the work of electrical equipment, and detection of the received signal take place.

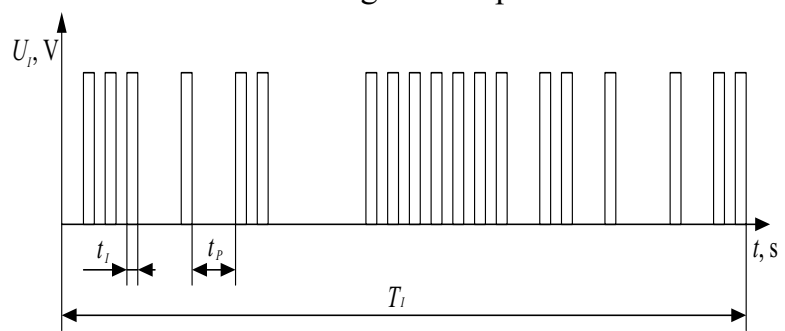

Figure 5. The signal after being detected in block 2

The signal after the detector is presented in Fig. 5. It consists of a random number of impulses having the same amplitude (voltage $U_{I}$ corresponds to the logical unit and depends on the system power - block 6) and the duration ( $t_{I}$ is the occurrence time of the impulse).The duration between impulses ( $t_{P}$ - pause time between impulses) is different, since electrical sparking or monotonic alteration of contact resistance is accidental. (Fig. 3).

From block 2 the signal is sent to the block of shaping the internal wiring fire risk alarm 3, in which the following logic of work is incorporated. Block 2 integrates all impulses over the measuring time $(\mathrm{Tm})$. This time can also be called the diagnostics time. The quantity $T_{I}$ depends on the contact time constant $\tau$, which is a function of contact mass:

$$
\tau \sim f\left(m_{c}\right)
$$

where $m_{c}$ is the contact mass. 
The analysis of the curves presented in Fig. 2 shows that $T_{I} \approx(5 \div 8) \tau$ (as over the time $\tau$ under continuous electric arcing or monotonic alteration of contact resistance the contact will be warmed up by $63 \%$ ), which corresponds to the calculated value of $\tau$ according to GOST 12.1.004-91 "Fire safety. General requirements" [19].

Therefore, if during the diagnostics $T_{I}$ the pause time between the impulses $t_{P}=0$, then the signal has a duty ratio of $Q=1$ (the maximum level of fire hazard of internal wiring). If during the diagnostics $T_{I}$ the pause time between the impulses $t_{P} \neq 0$, then the average duty ratio $\left(Q_{\text {avrg }}\right)$ of such a signal can be determined using the formula:

$$
Q_{\text {avrg }}=\frac{\sum_{1}^{n} t_{I}}{T_{I}}
$$

where $n$ is the quantity of impulses during time $T_{l}$, according to Fig. 5 .

This value indicates the level of fire hazard of internal wiring.

To realize such a work logic of the block 2, the following assumptions should be considered:

1) in the internal wiring, which is operated in real conditions, the amplitude of the impulses (Fig. 5) may be different due to different power and type of loads (active, active-inductive, active-capacitive, etc.). Therefore, the method of monitoring and diagnostics of wiring by means of the automatic system involves connecting predetermined resistive loads $\left(\cos \varphi=1\right.$ and $\left.\mathrm{P}_{L}=100 \mathrm{~W}\right)$, as that will allow one to detect a signal with the same amplitude of impulses;

2) the detection of signal in block 2 (Fig. 4) is unipolar and considers only the positive half-waves of the load current sine wave, so the signal needs to be doubled (intensified) to account the energy of the negative halfwaves, i.e. to reduce $\mathrm{Q}_{\text {avrg }}$ by half;

3) the adequacy of the device work is logically implemented provided that one spot of electrical sparking or monotonic alteration of contact resistance is present, whereas when there are two or more simultaneous emergency spots, the parallel superposition of signals from different sources should be taken into account to initiate high-frequency harmonic components.

Based on the foregoing, 5 levels of fire danger of internal wiring are embedded in block 2. The characteristics of the levels for total load $\mathrm{P}_{\mathrm{L}}=1400 \mathrm{~W}$ :

- Level 1 - electrical sparking or monotonic alteration of contact resistance is absent (technical inspection of internal wiring is not required);

- Level 2 - electrical sparking or monotonic alteration of contact resistance exists in the contact for 5 seconds with a pause $\left(t_{P}\right)$, which allows one to dissipate the produced heat into the environment (a technical inspection of the internal wiring and correction of the malfunction is necessary within a week);

- Level 3 - electrical sparking or monotonic alteration of contact resistance exists in the contact for 10 seconds with a pause $\left(t_{P}\right)$, which does not allow one to fully dissipate the produced heat into the environment (a technical inspection of the internal wiring and correction of the malfunction is necessary within a day);

- Level 4 - electrical sparking or monotonic alteration of contact resistance exists in the contact for 15 seconds with a pause $\left(t_{P}\right)$, which does not allow one to dissipate the produced heat into the environment (a technical inspection of the internal wiring and correction of the malfunction is necessary within 12 hours);

- Level 5 - electrical sparking or monotonic alteration of contact resistance exists in the contact for 20 seconds without any pause $\left(t_{P}\right)$ which would have allowed one to dissipate the produced heat into the environment (an immediate shutdown of the internal wiring of the building is required till the malfunction correction). In this case, the system is able to generate signals to disconnect automatic circuit breakers QF1-QF3 sent to the additional releases (not shown in Fig. 4).

Further, the signal of a fire hazard of internal wiring, formed in block 3 , is shown in the digital display of the levels of fire danger 4, which includes liquid-crystal screen to display the levels of fire hazard of the internal wiring. Starting from level 3 (high level), the block of sound indication of the levels of fire danger 5 turns on, and an audio signal takes place.

The entire automatic system of monitoring and diagnostics of the wiring 7 is powered by a power supply unit 6 , which is a portable lithium-ion battery with a possibility of recharge. Should the system 7 be used continuously on site, its powering can be done stationary.

To determine the emergency spots in the internal wiring one should disconnect consistently and systematically the active loads 10 and 12 . If the level of fire hazard is reduced or becomes acceptable after having disabled one of the active loads, this section of internal wiring must be subjected to preventive inspection for mechanical damage of wires and cables as well as defective or aged contacts.

If the installation of wires, cables and contacts was done thoroughly, then automatic system 7 shows the first level (low level) of fire hazard. Such wiring can be operated after the measurement of the resistance of its insulation in accordance with the rules for electrical installation [15].

The proposed automatic system of monitoring and diagnostics of electrical wiring is considered for implementation in applications of smart houses, smart grids other case studies of cyberphysical systems [20][25].

\section{Conclusion}

The suggested principle and scheme of functioning of the automatic system of monitoring and diagnostics of wiring allows one to assess the quality of the installation and the aging of wires, cables and power contact connections, which provide an acceptable level of wiring fire safety before putting it into service. Moreover, this eliminates the future possibility of formation of sources of ignition for insulation and other inflammable materials (dust, rags, 
etc.) due to the occurrence of electrical sparking or monotonic alteration of contact resistance in the wiring. This approach of monitoring and diagnostics does not require large expenditures of man-hours and can be performed by one installer, thereby reducing its cost and determining the economic efficiency of its use in real operating systems in smart environments and distributed robotic systems

This work is partially supported by the Russian Foundation for Basic Research (grant № 16-29-04101ofi_m).

\section{References}

1. N. Brushlinsky, M. Ahrens, S. Sokolov, P. Wagner. World Fire Statistics, Center of Fire Statistics of CTIF, 20: 63, (2015).

2. G.I. Smelkov. Fire safety of the electrical wiring, M: Kabel: 328, (2009).

3. S.V. Soleniy, O.Ya. Solenaya, A.P. Kovalev, G.V. Demchenko. Formation of algorithms of functioning of the automatic system of monitoring and diagnostics of electrical wiring, Information management systems, 2/75: 30-36, (2015).

4. A. Kovalyov, S. Soleniy, O. Solenaya. Development of electric current sensor for the device protecting electrical wiring against sparks, Material science forum, 856: 337-342, (2016).

5. Md. Manjur Ahmed, A.S.N. Huda, Nor Ashidi Mat Isa. Recursive construction of output-context fuzzy systems for the condition monitoring of electrical hotspots based on infrared thermography, Engineering Applications of Artificial Intelligence, 39: 120-131, (2015).

6. Qi Zi-bo, Gao Wei, Zhang Ying-cong. The Development of Electric Arc Fault Simulation Test Device, Procedia Engineering, 52: 297-301, (2013).

7. Qiongfang Yu, Dezhong Zheng, Yi Yang, Aihua Dong. An Arc Fault Detection Method Based on Wavelet Feature Extraction and the Design and Realization by LabWindows, CVI. Journal of computers, 8/2: 417-424, (2013).

8. Chi-Jui Wu, Yu-Wei Liu. Smart detection technology of serial arc fault on low-voltage indoor power lines, Electrical Power and Energy Systems, 69: 391-398, (2015).

9. Chen Nan, Tang Yixing, Zhang Qian. Experimental research about the current characteristic of the fault arc in series based on wavelet analysis, Procedia Engineering, 84: 736-741, (2014).

10. V. I. Gudim, U. I. Rudik, P. G. Stoliarchuk, V. M. Van'ko. Analyzer of resistance of two-wire electrical networks up to $1000 \mathrm{~V}$, Patent of invention 80325 Ukraine, № a200508664, 14: 8, (2007).
11. V. Torres, J.L. Guardado, H.F. Ruiz, S. Maximov. Modeling and detection of high impedance faults, Electrical Power and Energy Systems, 61: 163-172, (2014).

12. Chen Luping, Wang Peng, Xu Liangjun. Novel detection method for DC series arc faults by using morphological filtering, Electric Power Systems Research, 119: 91-99, (2015).

13. E. Tisserand, J. Lezama, P. Schweitzer, Y. Berviller. Series arcing detection by algebraic derivative of the current, The Journal of China Universities of Posts and Telecommunications, 22/5: 84-91, (2015).

14. J. Lezamaa, P. Schweitzera, E. Tisseranda, J.B. Humberta, S. Webera, P. Joyeux. An embedded system for AC series arc detection by inter-period correlations of current, Electric Power Systems Research, 129: 227-234, (2015).

15. Rules of installation for electrical devices (M.: publ «Knorus», Moskow), 488, 2014.

16. S.V. Soleniy. Increasing the fire safety of the facilities with eletrical networks of a current with voltage up to $1 \mathrm{~kW}$ (Autoref. dis. for applying for the degree Candidate of Engineering Sciences / UkrNIIPB. Kiev): 20, (2011).

17. O.Ya. Solenaya. Forecasting, monitoring and prevention of appearance of sources of ignition of combustible material in electrified areas (Autoref. dis. for applying for the degree Candidate of Engineering Sciences / DonNTU. Donetsk): 22, (2014).

18. V.A. Kazakov. Electrical appliances. A textbook for High schools (M.: publ. "RadioSoft"): 372, (2010).

19. GOST 12.1.004-91. Fire safety. General requirements (M.: Publ. of standards): 77, (1992).

20. R.M. Yusupov, A.L. Ronzhin. From Smart Devices to Smart Space, Herald of the Russian Academy of Sciences, 80/1: 45-51, (2010).

21. L.I. Chubraeva, A.L. Ronzhin, A.V. Shyshlakov, Al.L. Ronzhin, V.F. Shyshlakov. The Concept of Building Intelligent Systems Management for Protected Objects Decentralized Energy, SPIIRAS Proceedings, 33: 207-226, (2014).

22. V.P. Dashevsky, M.M. Bizin. Overview of onboard computers based SMARC-modules for robotic systems, Bulletin of TUSUR, 3/37: 91-97, (2015).

23. A.A. Karpov, A. Lale, A.L. Ronzhin. Multimodal assistive systems for a smart living environment. SPIIRAS Proceedings, 19: 48-64, (2011).

24. A.I. Motienko, A.L. Ronzhin, N.A. Pavlyuk. Modern developments of rescue robots: possibilities and principles of their application, Science Bulletin of the NSTU, 3/60: 147-165, (2015).

25. A.L. Ronzhin, V.Yu. Budkov. Multimodal Interaction with Intelligent Meeting Room Facilities from Inside and Outside, NEW2AN/ruSMART 2009, LNCS 5764: 77-88, (2009). 